\title{
Erkrankungen im Alter
}

$$
E^{p}
$$
pidemiologische Daten geben einen klaren Hinweis: die Anzahl älterer Menschen steigt und in der Folge werden Erkrankungen im Alter zunehmen. Neuropsychiatrische Krankheiten wie Depressionen, Demenzen, M. Parkinson und der Hirninfarkt sind im Alter häufig und gewinnen damit für das ärztliche Handeln immer größere Bedeutung. Umfassende Kenntnisse zu diesen Krankheiten sind für die drei Berufsgruppen, deren Titel gesamteuropäisch gelten, also Neurologen, Psychiater und Neuropsychiater, unverzichtbar. Mit diesem Themenheft hat sich psychoneuro zum Ziel gesetzt, relevante Erkenntnisse zu diesen Erkrankungen im Alter für die Praxis aufzuarbeiten.

Eva Grips und Georg Adler arbeiten medikamentöse und psychotherapeutische Ansätze bei der Behandlung der Depression heraus. Bei den medikamentösen Ansätzen werden dabei neuere Ansätze favorisiert. Dabei sollte nicht außer Acht gelassen werden, dass durchaus auch der Einsatz älterer Substanzen, wie z.B. Trazodon, auf Grund ihrer geringen Nebenwirkungen noch seine Berechtigung hat. Letztendlich entscheidet bei einem individuellen Patienten das Nebenwirkungsprofil über den Einsatz eines Medikamentes. Interessant ist in diesem Artikel auch die Darstellung eines in Mannheim entwickelten speziell auf ältere Menschen ausgerichteten verhaltenstherapeutischen Programms.

Matthias Löhle und Heinz Reichmann zeigen sehr differenziert und sachgerecht die aktuellen Diagnoseund Behandlungsmöglichkeiten bei M. Parkinson auf. Die Erkenntnisse münden in einen gut nachvollziehbaren Behandlungsplan, der eine wahre Hilfe für die Praxis sein sollte.

Christoph Baumsteiger erarbeitete umfassend die für Diagnose und Therapie des Hirninfarkts vorliegenden Fakten. Ein besonderer Schwerpunkt liegt dabei auf der Prävention, was für die Praxis sehr zu begrüßen ist.
Frölich et al. eröffnen einen sehr differenzierten Überblick zur Messung von Therapieerfolg mit dem Ziel, für den Patienten relevante Veränderungen abzubilden. Ein Bezug zu aktuellen Leitlinien wird hergestellt. Beispielhaft auch für die anderen Erkrankungen des Alters könnte die Diskussion um die Therapieerfolgsmessung sein. Für die Therapieerfolgsmessung stellen Frölich et al. die Wertigkeit des ärztlichen Urteils als die entscheidende Variable heraus. Nicht vergessen werden sollte aber, dass insbesondere bei Krankheiten, die primär Denken und Fühlen beeinträchtigen, auch dem Angehörigen- oder Pflegendenurteil eine hohe Bedeutung zukommt. Beide gewinnen deutlich, wenn qualitativ hochwertige Psychometrie sie untermauert. Nur ein geschulter Tester wird gültige Resultate erhalten. Nutzt er z.B. zur Verlaufskontrolle bei Demenz validierte Testverfahren wie ADAS-cog, SKT oder TFDD, wird seine Therapieerfolgsentscheidung auf eine solidere Basis gestellt.

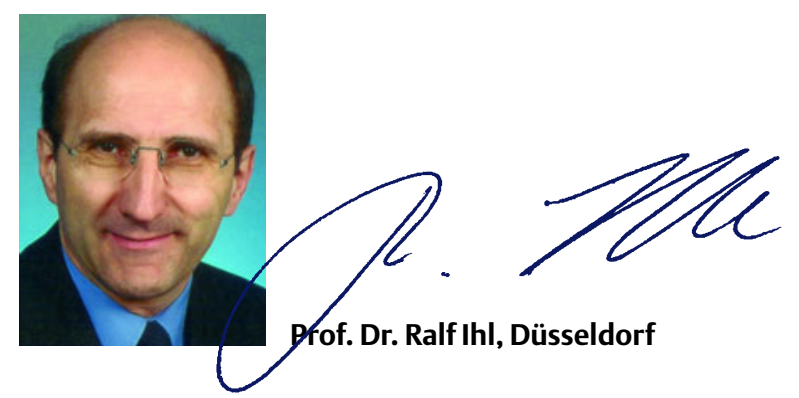

